Esta revista forma parte del acervo de la Biblioteca Jurídica Virtual del Instituto de Investigaciones Jurídicas de la UNAM

Rentería, Adrián, La tutela de los derechos en una sociedad democrática, México, Ediciones Coyoacán, 2014, 179 pp. ${ }^{1}$

En el libro La tutela de los derechos en una sociedad democrática,²Adrián Rentería reúne una serie de artículos sumamente interesantes, que de manera clara y analítica atienden temas propios de la filosofía jurídica y política. Temas como la noción de democracia, Estado de derecho, Constitución, elecciones, derechos humanos, entre otros. Mi objetivo en esta reseña es plantear algunas dudas que tengo en relación con el enfoque metodológico que Rentería adopta para atender algunos de estos temas, sobre todo el de la democracia y el fundamento de los derechos humanos; pero antes me gustaría, de manera muy breve y en unos cuantos párrafos, dar un panorama general del libro.

Son cuatro los capítulos del libro. En el primero, "En busca de una idea de democracia", Rentería somete la noción de democracia a un análisis conceptual interesante, sobre todo porque tiene claro los peligros que ello implica; por un lado, un concepto de democracia inundado de retórica, misma que es difícil de extraer del discurso político, que llena el contenido del concepto de ocurrencias y oportunismos, y puede que poco o mucho tengan que ver con la naturaleza de la democracia. Y por otro lado, por los problemas de ambigüedad y vaguedad del que adolece el concepto (p. 13). Rentería rápidamente se presenta como defensor de la democracia y defiende un concepto de democracia "sustancial", una democracia con sustento que ve hacia el contenido de las decisiones políticas, más que una noción formal de la misma. Para ello, el autor considera indispensable aclarar el concepto, junto con otros, como el de derechos humanos y Constitución. Esta noción de "democracia sustancial" es la que el autor defenderá a lo largo de su trabajo (pp. 35 y ss.).

En el capítulo segundo, "La difícil relación entre Constitución y democracia", Rentería apuesta por continuar con la defensa de su idea de democracia sustancial, y considera que esta forma correcta de explicar la democracia va de la mano del concepto de Constitución, pero no cualquier noción de Constitución, una noción también material de

\footnotetext{
${ }^{1}$ Agradezco los comentarios de Jesús Camarillo y Ricardo Reyes. El texto se benefició también de una discusión y presentación del libro en el Instituto de Investigaciones Jurídicas de la UNAM, donde participamos Adrián, Pedro Salazar, Guillermo Estrada y yo.

${ }^{2}$ De aquí en adelante sólo se mencionará el número de página en el texto al citar esta obra.
} 
Esta revista forma parte del acervo de la Biblioteca Jurídica Virtual del Instituto de Investigaciones Jurídicas de la UNAM

la Constitución, una que explica el contenido de derechos y que expone la validez del derecho mismo desde un enfoque sustantivo; es decir, que para Adrián, como para muchos otros autores, no basta con determinar el contenido del derecho a través de requisitos formales o hechos sociales constatables, como identificar las etapas del proceso legislativo o contar cuántos ministros aprobaron o no tal jurisprudencia. Más bien, lo que es derecho se determina a través de un análisis material del contenido de derechos humanos y principios de organización política que, incluso, resultan ser inmodificables (pp. 61 y 62). Adrián, en otras palabras, se une a una corriente contemporánea de filósofos del derecho que abogan por un Estado constitucional de derecho, más que un Estado de derecho legislativo, un Estado constitucional de derecho que se debe relacionar con la democracia sustancial, y así ubicar estos conceptos en su justa dimensión (pp. 63 y ss.).

Por su parte, el tercer capítulo es, desde mi punto de vista, más técnico. Se trata de un capítulo que si bien se atiende desde la filosofía política y jurídica, se inclina más hacia el derecho electoral. Esto, porque los temas propios del capítulo son los procesos para elegir a los que toman las decisiones en nuestra organización política y los métodos que se siguen para que los representantes tomen las decisiones. Así, Rentería discute los principios de mayoría y unanimidad en la toma de decisiones, y a través de estudios empíricos y de derecho comparado, resalta las complicaciones mexicanas de la baja representatividad que tienen las decisiones finales en política (pp. 90 y siguientes).

Finalmente, contamos con un capítulo cuarto, que bien podría denominarse “¿Quién le pone el cascabel al gato?", dado que trata la difícil tarea de argumentar una fundamentación de los derechos humanos, y cuyo título es "Sobre la justificación y la tutela de los derechos". Rentería, junto con otros autores contemporáneos, dicen: "yo le pongo el cascabel", y de manera muy interesante discute esta fundamentación a través de tres opciones, que para el autor existen, y se inclina por una fundamentación jurídica de los derechos humanos (pp. 144 y ss.), justificación que es uno de los puntos que quiero discutir en esta reseña.

Como lo mencioné al inicio, sólo me enfocaré a comentar dos puntos del libro de Rentería: el enfoque metodológico desde el que discute el análisis de democracia sustancial, y la fundamentación de los derechos humanos, temas que se argumentan en los capítulos uno, dos y cuatro. 
Esta revista forma parte del acervo de la Biblioteca Jurídica Virtual del Instituto de Investigaciones Jurídicas de la UNAM

Poco tengo que decir del capítulo tres, sólo una cuestión: cuando Rentería analiza los aspectos técnicos y electorales de la democracia, temas como la unanimidad y mayoría en la toma de decisiones (pp. 83-90), me hubiera gustado ver un análisis más profundo del germen de justicia que encontramos en la democracia y en la toma de decisiones por mayoría. Desde mi punto de vista, una discusión seria y profunda de este aspecto moral de la democracia y por qué los números mayoritarios importan moralmente, es lo único que puede rescatar a la democracia como principio de organización política de la crisis en la que se encuentra. Regresar las discusiones a este aspecto moral y político y examinar el resto de instituciones a la luz de este principio beneficiaría las metas a las que debemos aspirar hoy en día. Por ejemplo, visto el problema desde esta perspectiva, podemos pensar en eliminar o modificar aspectos de los partidos políticos, de la representatividad y de todas las instituciones que cobijadas bajo el manto de democracia se encuentran en tratamiento paliativo frente al ciudadano que justificadamente no tiene interés en votar y menos en participar.

\section{Dudas metodológicas}

Pero vamos a los puntos principales que quiero discutir del trabajo de Rentería. La pregunta básica que quiero plantear es la siguiente: ¿de dónde obtengo lo "sustancial" de la democracia? De dónde se extrae esta idea, que ayuda, tanto para acotar el concepto de democracia como para dar una explicación más acertada, según Rentería.

Primero el punto de la acotación del concepto. Recordemos que en el capítulo uno, Rentería desea evitar los problemas de ambigüedad y vaguedad del concepto, así como alejarse de toda la carga emotiva y retórica que implica el concepto de democracia. La idea del autor es que si nos ayudamos de otros conceptos, como el de Constitución y derechos fundamentales o derechos humanos, esto se logra. Pero de manera muy breve me gustaría resaltar que estos otros conceptos, de los cuales se ayuda Rentería, también adolecen de los mismos problemas. Considero que tanto el concepto de Constitución como el de derechos fundamentales también son dudosos en su aplicación. Por ejemplo, tengo serias dudas de si el concepto Constitución implica ciertas cláusulas intocables, como en algún punto lo argumenta Rentería (p. 62). ¿Cuál es el argumento importante para sostener una obligación tan fuerte? ¿El consenso, el contenido de esa Constitución? Y ¿por qué no podemos cambiar el núcleo de dicha Constitución para contar con mejores instituciones? Cambios que resulten de una discusión importante. En algún momento Rentería argumenta que los derechos humanos no son eternos ni inmutables, sino que 
Esta revista forma parte del acervo de la Biblioteca Jurídica Virtual del Instituto de Investigaciones Jurídicas de la UNAM

son producto de la voluntad humana (pp. 153 y 154), pues parece que aquí tenemos un ejemplo de contenido jurídico inmutable, donde la voluntad humana se encuentra limitada para modificarlo.

Por otra parte, sucede lo mismo con el concepto derechos fundamentales o derechos humanos; el concepto adolece también de vaguedad, y en algunos puntos su aplicación al caso concreto es dudoso. Los derechos, ¿son esos principios que argumenta Dworkin? ¿Son argumentos de principio que triunfan ante argumentos de directrices políticas? ${ }^{3} \mathrm{El}$ punto no es tan claro. Además, si un concepto está impregnado de retórica política y carga emotiva es el de derechos humanos, ya que unos Estados lo usan para legitimarse, y otros para restarles dicha legitimidad. Con esto no quiero decir que no se puede explicar un concepto dotado de vaguedad; esto es muy viable, pero no creo que se logre el objetivo de acotarlo nítidamente, como se lo propone Rentería al inicio del capítulo.

Por otra parte, y más interesante desde mi punto de vista, está el planteamiento de la misma pregunta que formulé — ¿de dónde obtengo lo sustancial de la democracia?-, pero vista desde la otra perspectiva; es decir, desde la parte de lo "sustancial" para explicar mejor la democracia. Voy a citar una parte clave del argumento del autor: “Como hemos visto, la concepción sustancial de la democracia tiene origen en una observación detallada de los fenómenos jurídicos y, por tanto, esta concepción puede ser vista como una visión en la que la dimensión normativa es imprescindible" (p. 50).

Esta idea de Rentería resulta importante ubicarla en su justa dimensión, ya que de ella depende su aportación principal. La preocupación inicial es la siguiente: ¿qué trabajo hace lo descriptivo y qué lo normativo en este argumento? Resulta obvio que no puedo extraer lo normativo de lo descriptivo; es decir, de la simple observación de los fenómenos jurídicos no obtengo normatividad, y de la simple observación de los fenómenos jurídicos no obtengo nada en la vecindad de una noción sustancial de la democracia ni de otro concepto. Para reiterar la idea común: no puedo deducir un deber ser del ser. Pero parece que este es el argumento del autor, cuando menciona que la concepción sustancial de la democracia tiene su origen en una "observación detallada de los fenómenos jurídicos".

\footnotetext{
3Véase en general, Los derechos en serio, Barcelona, Planeta-De Agostini, 1993.
} 
Esta revista forma parte del acervo de la Biblioteca Jurídica Virtual del Instituto de Investigaciones Jurídicas de la UNAM

Podemos esforzarnos para dar una interpretación más caritativa de lo que dice Rentería, en la que lo "sustancial" de la democracia se obtiene de la normatividad de lo jurídico, y en la que realmente la parte descriptiva es una forma de plantearlo, pero sin que se tome literalmente. En otras palabras, para el autor, lo sustantivo se obtiene de lo normativo del derecho. Así, no hay cruces indebidos, y se obtiene normatividad de otra normatividad. Pero aún así, tengo serias dudas de que la normatividad del derecho le proporcione a Rentería elementos para su argumento. Dejaré pendiente mi argumento en este sentido, porque se atenderá en la siguiente observación, y debo primero mencionar la siguiente idea del autor:

En el capítulo dos, Rentería insiste en extraer lo "sustancial" de la democracia de la observación de los fenómenos jurídicos, y lo plantea así:

Con democracia sustancial, Ferrajoli se refiere, y en este escrito sigo esta línea de razonamiento, a lo que debe ser, y no, por el contrario a lo que es. En esta noción se encuentran entrelazados, en forma muy estrecha, elementos de naturaleza política y jurídica, y ambos, como debiera sernos claro, tienen que ver decididamente no con lo que es, con lo que realmente sucede, sino que constituyen un ideal, un proyecto de vida. Este aspecto "ideal", de deber ser, es tal vez mayormente evidente en la esfera del derecho, cuya característica principal consisten en proponerse como un conjunto de prescripciones que tienen como objeto orientar nuestras acciones; lo que significa que el derecho no describe el mundo y nuestro comportamiento (si lo hiciera pertenecería a la esfera del ser), sino que trata de modificarlo (y por ello pertenece a la esfera del deber ser). Como hemos visto, la concepción sustancial de la democracia tiene origen en una observación detallada de los fenómenos jurídicos: en ella el derecho es quizá el elemento fundamental. Y por ello esta concepción puede ser vista como una visión en la que la dimensión normativa es imprescindible (p. 72).

Mencionar esta cita tan larga resulta indispensable, porque abona en favor de nuestra interpretación más caritativa mencionada anteriormente. Es decir, si bien Rentería insiste en mencionar la observación detallada de los fenómenos jurídicos para extraer lo "sustantivo" de la democracia, más bien se refiere a que esta sustantividad se obtiene de la sustantividad y normatividad del derecho mismo.

Una cuestión preliminar en este sentido: al leer estos dos capítulos del libro yo pensaría que el autor extraería lo "sustantivo" de elementos morales y políticos que se 
Esta revista forma parte del acervo de la Biblioteca Jurídica Virtual del Instituto de Investigaciones Jurídicas de la UNAM

preguntan sobre la legitimidad moral y lo benéfico de contar con un sistema democrático y por qué este sistema es moralmente deseable. Pero el autor me cambió totalmente el panorama; prácticamente me pide que no busque más allá, que el derecho mismo goza de esta normatividad y que ella sirve para darle normatividad a conceptos políticos y morales tan importantes como democracia. Yo pensaría, reitero, que la perspectiva era a la inversa: buscar en lo moral dicha sustantividad para la democracia. El argumento de Rentería me puso a pensar.

Desafortunadamente, aún no estoy convencido. Pensar que conceptos políticos y morales, en este caso la democracia, son parasitarios de la normatividad del derecho, resulta extremadamente dudoso; creo que el objeto está en la otra mano, y Rentería elige el lugar equivocado.

Reflexionemos un poco en relación con esta tesis de Rentería: ¿de dónde obtienen sentido conceptos como "deber", "tener que”, “obligación” en el campo jurídico? Por ejemplo, yo tengo que respetar la propiedad de mi vecino, debo separar la basura, tengo una obligación de pagar impuestos. Otra forma de plantearlo es: ¿de dónde obtengo la normatividad en el derecho? Y éstas son las preguntas que nos debe responder Rentería antes de dar por concluido el argumento; en caso contrario, el autor está ofreciendo algo que no tiene.

El autor cuenta con varias opciones; una puede radicar en aceptar la norma básica del sistema jurídico; ${ }^{4}$ o bien en sostener que es la práctica social de ciertas reglas lo que le da su normatividad; 5 etcétera; pero aun cuando Rentería proporcione un argumento sólido en relación con cómo la normatividad del derecho se queda en el derecho mismo —argumento sólido que dudo exista—, no tenemos elementos para llegar a la siguiente conclusión: que la normatividad del derecho es tan importante como para trasladarse y dar sentido a otras normatividades en lo político y moral.

Cómo lo mencioné, para mí la normatividad del derecho es parasitaria de otras, y no a la inversa, concretamente de la normatividad moral. Esto no significa que las obligaciones jurídicas siempre reúnen los exigencias de una obligación moral, pero se

\footnotetext{
4El lugar clásico es Kelsen, Teoría pura de/ derecho, México, Porrúa, 1991.

${ }^{5}$ En este caso el clásico de Hart, El concepto de derecho, trad. de Genaro Carrió, Buenos Aires, AbeledoPerrot, 1995.
} 
Esta revista forma parte del acervo de la Biblioteca Jurídica Virtual del Instituto de Investigaciones Jurídicas de la UNAM

presentan como si fueran morales por una autoridad que por lo menos pretende ser 0 bien es una autoridad legítima moral. 6 o nos podemos ir al otro extremo, que no comparto: las obligaciones jurídicas, salvo casos extremos y concretos, siempre son obligaciones morales y emitidas por autoridades legítimas morales. ${ }^{7} \mathrm{O}$ adoptar la noción dworkiniana contemporánea: el derecho es una rama de la moralidad política y los derechos jurídicos simplemente son derechos morales ejecutables ante los tribunales. ${ }^{8}$ En cualquier caso, los argumentos de Rentería tienen que atender esta otra perspectiva que curiosamente resulta ser la principal en la filosofía jurídica actual, independientemente de la corriente a la cual pertenezcamos. Una perspectiva que insiste en que estos conceptos de "debe", "tener que" y “obligación" en lo jurídico, se tienen que relacionar de alguna u otra forma con una normatividad adicional, para ser precisamente "normativa". 9

\section{Justificación de los derechos humanos}

Así, llegamos al último punto que me llamó mucho la atención del libro de Rentería, uno que amerita discusiones importantes. Se trata del difícil tema de la justificación de los derechos humanos. Al respecto, el texto inicia con tres posibles vías que tenemos para justificar los derechos humanos: una justificación sociohistórica, que analiza las sociedades y su desarrollo y cómo se han ido generando estos derechos humanos en diferentes sistemas jurídicos (p. 143); una justificación jurídica, donde se justifica por qué están reconocidos en las cartas constitucionales, en tratados y convenciones internacionales (p. 143 ), y una justificación ético-política, que argumenta la justificación de los derechos en razón de su valor, en tanto que dichos derechos reflejan determinados valores morales (p. 143).

Coincido con Rentería en que la primera opción debemos descartarla rápidamente. Veo difícil justificar algo con el simple hecho histórico de que así ha sucedido a lo largo

\footnotetext{
${ }^{6}$ Sigo a Raz en este sentido véase sobre todo Ethics in the Public Domain, Oxford, Clarendon Press, 1994.

${ }^{7}$ Podemos aquí pensar en Finnis, véase Natural Law and Natural Rights, Oxford, Clarendon Press, 1980.

8Justice for Hedgehogs, Cambridge, Harvard University Press, 2011, pp. 405 y 407. (Hay traducción al castellano en México por el Fondo de Cultura Económica, 2014).

${ }^{9}$ Claro, lo interesante radica en la explicación de esta relación con la normatividad moral.
} 
Esta revista forma parte del acervo de la Biblioteca Jurídica Virtual del Instituto de Investigaciones Jurídicas de la UNAM

del desarrollo de los sistemas jurídicos; esto puede explicar los derechos humanos, pero no justificarlos.

El argumento del autor también descarta la tercera vía, es decir, la ético-política, debido a las serias dudas que tiene Rentería de las premisas en las que descansan sus tesis; o sea, una idea de valores absolutos y universales de nociones inmutables y definitivos acerca de ciertos principios morales.

El argumento finalmente es uno de justificación de los derechos humanos en lo jurídico. El argumento se presenta de la siguiente manera:

La presencia de los derechos, en otras palabras, se debe no a otra cosa que no sea la voluntad humana de incluirlos en la tabla de valores que rige los destinos de la sociedad. Los derechos, en este orden de ideas, no son el resultado de una ley natural por universal e inmutable que pueda considerarse; ni son tales en razón de una (verdadera o sólo presunta) naturaleza humana. Desde la perspectiva de la justificación jurídica el anclaje de los derechos en la voluntad humana de plasmarlos en los textos mediante los cuales se disciplina el comportamiento de las personas y el funcionamiento de las estructuras sociales, es suficiente para justificar su presencia, sin necesidad de recurrir a factores diferentes (p. 154).

De nuevo tengo dudas en darle al derecho este lugar tan importante en nuestras vidas y en nuestros recuentos de lo que es el derecho, ahora en el tema de la justificación de los derechos humanos. Para continuar la discusión de la fundamentación de los derechos humanos en la dimensión ético-política, podemos pensar en una alternativa que no descansa en las premisas que tanto incomodan a Rentería. Podemos discutir, por ejemplo, la respuesta de Rawls, ${ }^{10}$ y ahora Raz, ${ }^{11}$ de dar una explicación política de los derechos humanos, derechos que establecen límites a la soberanía de los Estados (cuya violación desarticula el argumento de que un Estado soberano puede hacer lo que quiera) y donde las violaciones a los derechos humanos posibilita tomar acciones en la arena internacional contra el Estado agresor.

Es una justificación político-moral, sin duda porque tengo buenas razones morales para darle reconocimiento institucional a estos derechos y limitar la soberanía, pero se

\footnotetext{
10 The Law of Peoples, Cambridge, Harvard University Press, 1999.

11“Human Rights Without Foundations”, Oxford Legal Studies Research Paper, núm. 14, 2007.
} 
atiende una de las principales preocupaciones del autor: dar una explicación contingente y no universal de por qué tenemos estos derechos; esto, porque depende del reconocimiento institucional, no de la naturaleza humana o de premisas dudosas de cierta vertiente del derecho natural. En otras palabras, para Rentería sigue abierta la tercera opción de una justificación ético-política que coincide con sus preocupaciones, sobre todo porque no creo que la justificación jurídica pueda llegar muy lejos. Las razones ya las mencioné al explicar mi escepticismo a la idea independiente de la normatividad del derecho, y por consecuencia a la importancia que se le asigna al derecho mismo.

Juan VEGA GómEZ12

${ }^{12}$ Investigador en el Instituto de Investigaciones Jurídicas de la UNAM. 